\title{
Joint Coordination During Whole-Body Lifting in Women With Low Back Pain After Pregnancy
}

\author{
Dianne A. Commissaris, PhD, Lena B. Nilsson-Wikmar, RPT, Jaap H. van Dieën, PhD, Helga Hirschfeld, PhD
}

ABSTRACT. Commissaris DA, Nilsson-Wikmar LB, van Dieën JH, Hirschfeld H. Joint coordination during whole-body lifting in women with low back pain after pregnancy. Arch Phys Med Rehabil 2002;83:1279-89.

Objective: To quantify differences in the kinematics of lifting between women with low back and/or pelvic pain after pregnancy and women without.

Design: Comparison study.

Setting: Research laboratory.

Participants: Volunteer sample of 7 women with pain (positive pain drawing, no physical examination) and 9 female controls (not matched).

Interventions: Not applicable.

Main Outcome Measures: Duration of downward and upward phases, relative instant of box lift-off, joint angles, spatial angles of trunk and pelvis, pelvic angle relative to trunk, and phase angle relationships between joints.

Results: The duration of the upward phase \pm standard deviation was longer in the pain group $(1731 \pm 290 \mathrm{~ms}$ vs $1489 \pm 187 \mathrm{~ms}, P=.031)$. At box lift-off, this group had less hip joint flexion $\left(101.9^{\circ} \pm 20.8^{\circ}\right.$ vs $\left.78.7^{\circ} \pm 12.4^{\circ}, P=.015\right)$ but more backward pelvis tilt relative to the trunk, that is, more lumbar spine flexion $\left(126.3^{\circ} \pm 16.8^{\circ}\right.$ vs $\left.109.0^{\circ} \pm 12.3^{\circ}, P=.031\right)$. The pain group showed an immediate transition from lumbar spine flexion to extension, whereas the controls maintained peak flexion for about $600 \mathrm{~ms}$. The peak phase lag between knee and hip joint extension in the upward phase was larger for the pain group $\left(-29.7^{\circ} \pm 8.3^{\circ}\right.$ vs $\left.-17.2^{\circ} \pm 5.5^{\circ}, P=.003\right)$.

Conclusion: Women with low back and/or pelvic pain after pregnancy showed different kinematics of lifting. Further research is needed to determine the exact relationship between the altered kinematics and the underlying disorder.

Key Words: Lifting; Low back pain; Pelvis; Pregnancy; Rehabilitation; Women.

(C) 2002 by the American Congress of Rehabilitation Medi-

From the Amsterdam Spine Unit, Institute for Fundamental and Clinical Human Movement Sciences, Faculty of Human Movement Sciences, Vrije Universiteit Amsterdam, Amsterdam, The Netherlands (Commissaris, van Dieën); Motor Control and Physical Therapy Research Laboratory, Neurotec Department, Division of Physiotherapy, Karolinska Institutet Stockholm, Stockholm, Sweden (Nilsson-Wikmar, Hirschfeld); and TNO Work and Employment, Hoofddorp, The Netherlands (Commissaris).

Supported by the Institute for Fundamental and Clinical Human Movement Sciences, Amsterdam; the Stichting Anna Fonds, Leiden, The Netherlands; the WennerGren Center Foundation for Scientific Research, Stockholm, Sweden; the Swedish Foundation for Health Care Sciences and Allergy Research, Karolinska Institutet, Stockholm, Sweden; and the Vardal Foundation, Stockholm, Sweden (grant no. V96159).

Presented in part at the 13th Congress of the International Society of Posture and Gait, June 1997, Paris, France, and at the 13th International Congress for Physical Therapy, June 1999, Yokohama, Japan.

No commercial party having a direct financial interest in the results of the research supporting this article has or will confer a benefit upon the author(s) or upon any organization with which the author(s) is/are associated.

Reprint requests to Dianne A. Commissaris, PhD, TNO Work and Employment, PO Box 718, 2130 AS Hoofddorp, The Netherlands, e-mail: d.commissaris@arbeid.tno.nl. 0003-9993/02/8309-6871 \$35.00/0

doi:10.1053/apmr.2002.33641 cine and the American Academy of Physical Medicine and Rehabilitation

$\mathbf{T}$ HE EXISTENCE OF back pain during pregnancy has been known since ancient times. There is a general impression that it has increased during the last decade; studies from Sweden and Great Britain have reported a prevalence of back pain during pregnancy of approximately $50 \% 1,2$ to $75 \% .^{3}$ Östgaard et al, ${ }^{2}$ for instance, found a 9 -month-period prevalence of $49 \%$ in 855 pregnant women studied from the 12th week of pregnancy until childbirth. Because $22 \%$ of the women had back pain at the 12 th week, the 6-month incidence was $27 \%$. For $36 \%$ of the cases, back pain was a severe problem, compromising normal, everyday life. In a follow-up study, ${ }^{4}$ on average 18 months after delivery, $30 \%$ of the women who completed the follow-up questionnaire stated that they still had some back pain and $7 \%$ reported having severe pain. Despite the high prevalence, the concept of back pain during and after pregnancy is not well defined,5,6 and the exact origin is often unknown.

Although a large number of epidemiologic studies into pregnancy-related back pain have been conducted during the last decade, few studies have addressed the question of how back pain after pregnancy affects the performance of daily activities, especially those that involve the back. However, we do know that chronic low back pain (LBP) patients in general show deficits in reaction time, coordination, and postural control. , $^{8}$ Also, reduced trunk muscle force and endurance have been reported. ${ }^{9-11}$ Thus, it can be expected that the performance of physically demanding tasks involving the back is affected in women who have back pain after pregnancy. In the present study, we investigated the performance of a bimanual, wholebody lifting task in women with and women without LBP after a pregnancy. By LBP after pregnancy we refer to pain in the area of the lumbar spine and/or pelvic girdle that arose during pregnancy and was still present at the time of our study (on average, 5mo after childbirth). We chose to study a bimanual whole-body lifting task because it is a physically demanding task involving the back, because it is performed on a daily basis by women with small children and because bending forward was reported to be painful by many women with pelvic pain after pregnancy. ${ }^{12}$

Our aims were to identify and quantify differences in the coordination of bimanual load lifting performed by women with and without LBP after pregnancy. We anticipated that the kinematics of this task would provide insight into the relationship between LBP and any impaired task performance. We analyzed joint angles, spatial angles of trunk and pelvis, and the pelvic tilt in the body. We also quantified the interjoint coordination by calculating the phase angle relationships between various joints. ${ }^{13}$ This method appropriately takes into account that the bimanual, whole-body lifting task is a complex multijoint movement. ${ }^{14}$ Previous studies ${ }^{13,14}$ on men have shown a consistent distal-to-proximal sequence of joint rotations during the phase of lifting the object, that is, the ankle joint was the first joint to begin extension followed by successive extension of the knee, hip, and lumbosacral joints. This sequence proved 
to be fairly fixed, but the timing depended on task factors, such as object mass, ${ }^{15,16}$ and personal characteristics, such as fatigue. ${ }^{17,18}$ In women, a distal-to-proximal sequence of joint extension was also found for the knee and hip joints. ${ }^{19}$

In the present study, we tested 3 hypotheses: (1) women with LBP would perform the upward lifting motion more slowly than the controls because trunk extension velocity is usually lower in patients with back pain ${ }^{20,21} ;(2)$ back pain-associated differences in the timing of the distal-to-proximal sequence of joint rotations during the phase of lifting would appear, analogous to the changes in timing, that are associated with changes in object mass and fatigue of the subject lifting ${ }^{15-18}$; and (3) women with back pain would stabilize the pelvis in the body and limit flexion in the lumbar spine because strain on injured passive structures is known to induce pain. ${ }^{22,23}$ This hypothesis is in accordance with the results of Larivière et al. ${ }^{24}$

\section{METHODS}

\section{Participants}

Nine clinically healthy women (NBP group) and 7 women with back pain (BP group) participated on a voluntary basis. All subjects were recruited from an ordinary postpregnancy exercise class, although not necessarily the same class. Because the purpose of the exercise class was to get fit again after a pregnancy, the program was adjusted to the physical condition and fitness level of women who recently underwent a pregnancy. The program did not incorporate any information about safe lifting techniques nor was it specifically aimed at back pain. The subjects first received verbal information about the study from the exercise instructor who was not involved in the study. Women who volunteered to participate then received detailed written information from one of the experimenters. All volunteers chose to participate. The inclusion criteria were (1) a delivery within 1 year before study (2) ongoing back and/or pelvic pain or (3) no ongoing back and/or pelvic pain. No exclusion criteria were applied, implying that subjects who had back pain before their last pregnancy were included. Subjects were assigned to 1 of the 2 groups (NBP, BP) by an author (LBN-W, a physical therapist) based on pain drawings and a medical history questionnaire. No physical examination was conducted. Subjects with a positive pain drawing in the low back and/or pelvic region were assigned to the BP group. BP and NBP subjects were not matched. One experimenter was aware of the type of subject (BP or NBP) entering the laboratory but the others were not. Because all experimental and analytic procedures were standardized and mostly automatic, the nonblinded experimenter seemed unable to influence the final data of individual subjects.

Characteristics of both groups are summarized in table 1 , whereas specific features of the back pain group are in table 2 . The BP subjects were examined on average 5.7 months after delivery. Their median assessment of pain was $27 \mathrm{~mm}$ on a visual analog scale (VAS; 0mm: no pain; 100mm: worst imaginable pain). Back pain onset for 2 subjects was between gestation weeks 1 and 12; for 3 subjects it was between weeks 12 and 24; for 1 subject between weeks 25 and 40; and for 1 other subject at delivery. Six of 7 subjects had also experienced episodes of back pain before their last pregnancy. In the BP group, the median value of the Disability Rating Index (DRI) over all 12 items was $29 \mathrm{~mm}$ on a VAS (0mm: without difficulty; 100mm: not capable at all). ${ }^{25}$ The task of lifting heavy objects received the highest rating; the median value was $58 \mathrm{~mm}$. The NBP subjects were examined on average 5.9 months after delivery. None reported pain at the time of the experiment, although 1 subject had experienced back pain during her last pregnancy, starting between gestation weeks 25 and 40 . No subject reported a history of back pain before her last pregnancy. The median values of both the total DRI and the ability to lift a heavy object were $0 \mathrm{~mm}$. The local ethics committee approved the study. All subjects received written information and gave their informed consent.

\section{General Procedure and Experimental Protocol}

The lifting task consisted of lifting a box $(360 \times 360 \times$ $250 \mathrm{~mm}$ ) weighing $8.3 \mathrm{~kg}$ with both hands. The subjects stood upright in bare feet with each foot on a force platform. The experimental setup is shown in figure 1, including the location of 14 reflecting markers. The subject was cued by an auditory signal to bend down and grip the 2 handles mounted on each side of the box (210mm above the bottom) and to lift the box to chest height. The task ended when the subjects stood in the upright position holding the box. Thereafter, the experimenter placed the box back on the floor. Each trial had a recording time of 5 seconds, and the movement was repeated 7 times. Before the experiments, each subject was able to practice the lifting task as many times as she wanted. No specific instructions were given regarding lifting technique and speed, and no restrictions were imposed on the initial position. Foot placement was prescribed by the size and position of both forceplates; the 2 plates (width, 203mm; length, $457 \mathrm{~mm}$ ) were positioned parallel, and the mediolateral distance between both long sides of the plates was $40 \mathrm{~mm}$. Problems with occlusion of the markers at the hip and on anterior superior iliac spine (ASIS) by the arm made it necessary to ask the subjects to keep the arms crossed in front of the body during initial standing. To facilitate the attachment of markers directly on the skin, the subjects wore a brassiere and briefs only.

\section{Apparatus, Data Acquisition, and Data Processing}

A 2-camera opto-electronic system ${ }^{\mathrm{a}}$ recorded the positions of 14 passive light-reflecting markers. ${ }^{26}$ Signals were digitized with an analog-to-digital converter at a frequency of $100 \mathrm{~Hz}$. Sampling time started $500 \mathrm{~ms}$ before the auditory signal that cued the subject to initiate the task. The digitized data were stored for further processing. Under the prevailing experimental conditions, the explored field was $2 \times 2 \mathrm{~m}$ and the accuracy $0.8 \mathrm{~mm}$. Thirteen hemispherical markers (diameter, 20mm) were attached to anatomic landmarks on the left side of the body, except for 3 markers that were put on the spinal column. The markers were attached at the following locations (see fig 1): (1) the mandibular joint, as close to the ear as possible; (2) the cheek close to the corner of the mouth; (3) the spinous process of the seventh cervical vertebra; (4) the spinous process of the 12th thoracic vertebra; (5) the spinous process of the fifth lumbar vertebra; (6) the ASIS; (7) the top of the greater trochanter; (8) just below the top of the lateral femoral condyle; (9) the top of the lateral malleolus; (10) the lateral side of the calcaneus; (11) the fifth metatarsophalangeal joint; (12) the acromion; and (13) the lateral epicondyle of the humerus. An additional marker (14) was placed on the box. The 3 markers on the spinal column were spherical and glued to the top of a plastic cone $(35 \mathrm{~mm}$ long) to ensure detection by the cameras. The 13 body markers defined the position of 11 body links (see fig 1). Links relevant for the present analyses were trunk (3-5), pelvis (6-7), L5-ASIS (5-6), upper legs (7-8), lower legs (8-9), and feet (10-11). Angles of the ankle, knee, hip, and sacral joints were calculated from the angle between 2 intersecting links: the ankle joint angle between links 8-9 and 10-11, the knee joint angle between $7-8$ and $8-9$, the hip joint angle between 6-7 and 7-8, and the lumbosacral joint angle between 3-4 and 5-6. Note that both the ankle and lumbosa- 
Table 1: Characteristics of the BP and NBP Groups

\begin{tabular}{|c|c|c|}
\hline & $\mathrm{BP}(\mathrm{n}=7)$ & $\operatorname{NBP}(n=9)$ \\
\hline Mean age $\pm S D(y)$ & $33.4 \pm 3.6$ & $34.0 \pm 3.4$ \\
\hline Mean weight \pm SD $(\mathrm{kg})$ & $62.0 \pm 8.7$ & $64.2 \pm 7.6$ \\
\hline Mean height $\pm \mathrm{SD}(\mathrm{m})$ & $1.67 \pm 0.03$ & $1.68 \pm 0.03$ \\
\hline Mean body mass index $\pm \mathrm{SD}\left(\mathrm{kg} / \mathrm{m}^{2}\right)$ & $22.3 \pm 3.0$ & $22.9 \pm 2.9$ \\
\hline Mean time since latest delivery $\pm \mathrm{SD}(\mathrm{mo})$ & $5.7 \pm 1.4$ & $5.9 \pm 1.3$ \\
\hline \multicolumn{3}{|l|}{ Previous pregnancies (no. of subjects) } \\
\hline 0 & 2 & 4 \\
\hline 1 & 0 & 2 \\
\hline 2 & 3 & 2 \\
\hline $3+$ & 2 & 1 \\
\hline \multicolumn{3}{|c|}{ Physical activity during leisure before pregnancy ( $n$ ) } \\
\hline Not at all & 2 & 4 \\
\hline Once a week & 4 & 3 \\
\hline Twice or more weekly & 1 & 2 \\
\hline \multicolumn{3}{|c|}{ Leisure time physical activity during pregnancy $(n)$} \\
\hline Not at all & 3 & 3 \\
\hline Once a week & 2 & 3 \\
\hline Twice or more weekly & 2 & 3 \\
\hline \multicolumn{3}{|l|}{ Leisure time physical activity now (n) } \\
\hline Not at all & 0 & 0 \\
\hline Once a week & 7 & 9 \\
\hline Twice or more weekly & 0 & 0 \\
\hline \multicolumn{3}{|l|}{ Experience of general fitness $(n)$} \\
\hline Good & 3 & 5 \\
\hline Moderate & 1 & 3 \\
\hline Bad & 3 & 1 \\
\hline Back pain during last pregnancy $(\mathrm{n})$ & 7 & 1 \\
\hline \multicolumn{3}{|l|}{ Back pain onset during last pregnancy $(n)$} \\
\hline Between gestation wk 1-12 & 2 & 0 \\
\hline Between gestation wk $12-24$ & 3 & 0 \\
\hline Between gestation wk $25-40$ & 1 & 1 \\
\hline At delivery & 1 & 0 \\
\hline Back pain before last pregnancy $(n)$ & 6 & 0 \\
\hline $\mathrm{DR} \mathrm{I}^{25}$ median (range) (in $\mathrm{mm}$ )* & $29(10-69)$ & $0(0-0)$ \\
\hline \multicolumn{3}{|l|}{ The $12 \mathrm{DRI}$ items, median (range) } \\
\hline Dressing (without help) & $5(0-47)$ & $0(0-0)$ \\
\hline Outdoor walks & $0(0-32)$ & $0(0-0)$ \\
\hline Climbing stairs & $23(3-40)$ & $0(0-0)$ \\
\hline Sitting for a longer time & $48(0-71)$ & $0(0-0)$ \\
\hline Standing bent over a sink & $48(0-100)$ & $0(0-0)$ \\
\hline Carrying a bag & $48(14-98)$ & $0(0-0)$ \\
\hline Making a bed & $13(0-100)$ & $0(0-0)$ \\
\hline Running & $24(8-100)$ & $0(0-0)$ \\
\hline Light work & $10(0-67)$ & $0(0-0)$ \\
\hline Heavy work & $28(0-100)$ & $0(0-0)$ \\
\hline Lifting heavy objects & $58(22-100)$ & $0(0-0)$ \\
\hline Participating in exercise/sports & $13(0-100)$ & $0(0-0)$ \\
\hline
\end{tabular}

* VAS scale: $0 \mathrm{~mm}$ (without difficulty) to $100 \mathrm{~mm}$ (not capable at all).

cral joint are not actual joints but junctions defined between the projection of 1 link onto another link. In all cases, extension was defined as an increase in angle. Spatial angles of pelvis and trunk were calculated relative to the horizontal axis of the ELITE coordinate system. ${ }^{\text {a }}$ Backward rotation of a segment in space was defined as an increase in angle. Pelvic tilt in the body was defined as the pelvic angle calculated relative to an axis perpendicular to the cephalocaudal trunk axis. A backward pelvic tilt in the body, coinciding with lumbar spine flexion, was defined as an increase in angle. Angles were low-pass filtered with a digital filter (zero phase lag, $3 \mathrm{~Hz}$, second-order Butterworth filter) and angular velocities were obtained by numeric differentiation (Lanczos 5-point differentiation filter) of the angle time series.

Electromyograms and ground reaction forces were recorded but not analyzed in the present article.

\section{Relative Phase Angle Analysis}

Phase angles were calculated according to Kelso et al. ${ }^{27}$ Joint angles and angular velocities were normalized such that the maximum value of each angle and angular velocity in a trial was set to 1 and the minimum value to -1 . Subsequently, a phase plot was constructed, with the angle on the ordinate plotted against angular velocity on the abscissa. The phase 
Table 2: Pain Characteristics in BP Group

\begin{tabular}{lc}
\hline & $\mathrm{BP}(\mathrm{n}=7)$ \\
\hline $\begin{array}{l}\text { Back pain rating at the experiments, median } \\
\text { (range) (mm)* }\end{array}$ & $27(2-98)$ \\
Back pain rating at worst, median (range) (mm)* & $81(65-100)$ \\
Time of worst back paint $(\mathrm{n})$ & 3 \\
In the morning & 1 \\
In the afternoon & 4 \\
In the evening & 2 \\
During the night & \\
Back pain changes since its last onset $(\mathrm{n})$ & 0 \\
Much worse & 4 \\
Somewhat worse & 3 \\
Unchanged & 0 \\
Somewhat improved & 0 \\
Very improved & \\
\hline
\end{tabular}

* VAS scale: $0 \mathrm{~mm}$ (no pain) to $100 \mathrm{~mm}$ (worst imaginable pain). † More than 1 choice allowed.

angle is the angle between the vector from the origin to a point on the phase plot and the right-hand ordinate. The relative timing of joint rotations was quantified for each trial by means of the relative phase of (adjacent) joints, ie, the phase differences between rotations in the ankle and knee joint, knee and hip joint, hip and lumbosacral joint, ${ }^{13,14}$ and ankle and lumbosacral joint. In all cases, the phase angle of the distal joint was subtracted from the phase angle of the proximal joint.

\section{Definition of Task Phases}

The lifting task was divided into 2 phases: the downward phase and the upward phase. The onset of the downward phase was defined $100 \mathrm{~ms}$ before the first sample at which the angular velocity of the knee joint clearly deviated from zero in a negative (flexing) direction. The end of the upward phase was defined $100 \mathrm{~ms}$ after the first sample at which the angular velocity of the knee joint returned to zero from positive (extending) values. Both samples were manually marked in the angular velocity time series by means of interactive data anal-

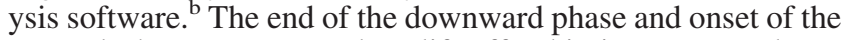
upward phase was set at box lift-off. This instant was determined from the onset of the vertical displacement of the box marker.

\section{Data Analysis and Statistics}

For each subject, the kinematic data of the first 5 trials without missing markers were further analyzed. For $1 \mathrm{NBP}$ subject and $1 \mathrm{BP}$ subject, we only had 3 suitable trials at our disposal because missing markers prevented us from obtaining all relevant angles. (We did not use a specific trial if we did not have the full set of angles.) To enable averaging of selected parameters within subjects and within groups, the time series of these parameters were normalized by means of cubic spline interpolation, setting the length of a downward and successive upward phase to $100 \%$ (onset down at $0 \%$, end up at $100 \%$ ). To identify and quantify differences between both groups, time series of the defined angles, angular velocities, and relative phase angles were first averaged within each subject (5 or 3 trials) and then averaged within each group (9 NBP subjects, 7 $\mathrm{BP}$ subjects). The same procedure was applied to determine mean values for total movement time, duration of downward phase, duration of upward phase, relative instant of box lift-off, baseline angle, lift-off angle, range of motion (ROM), angular velocity at lift-off, peak angular velocity in the down- and upward phases, and mean and peak relative phase angle in the down- and upward phases.
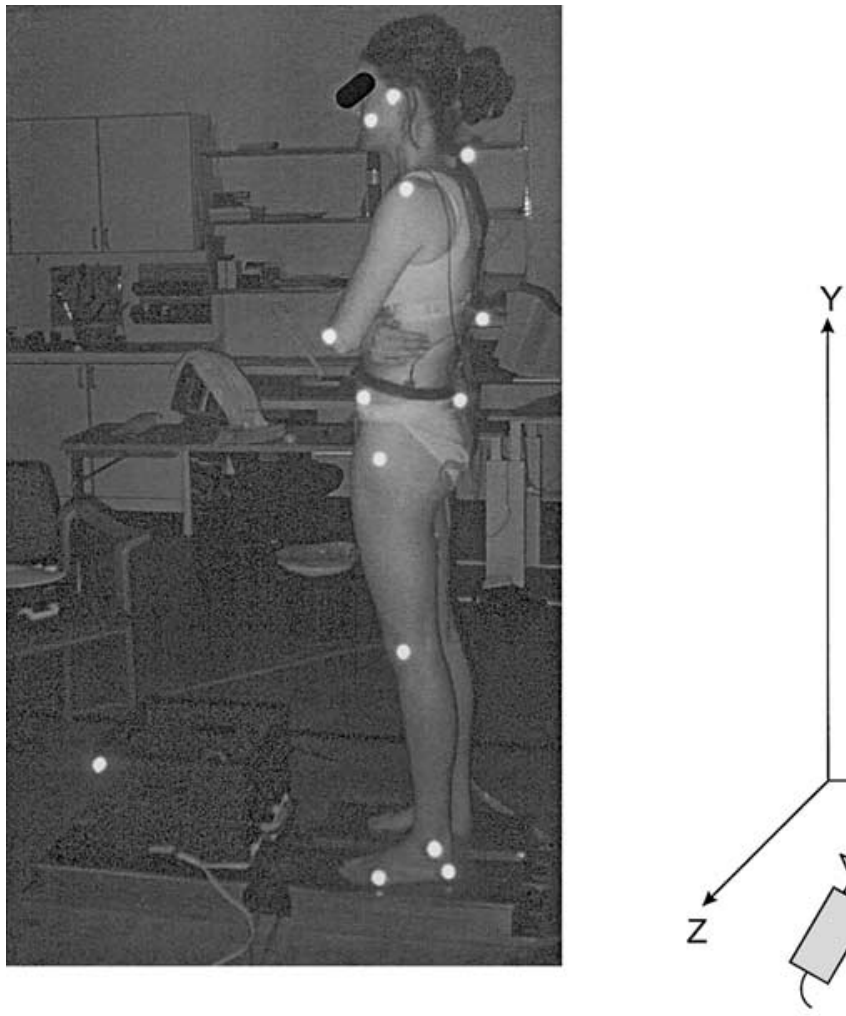

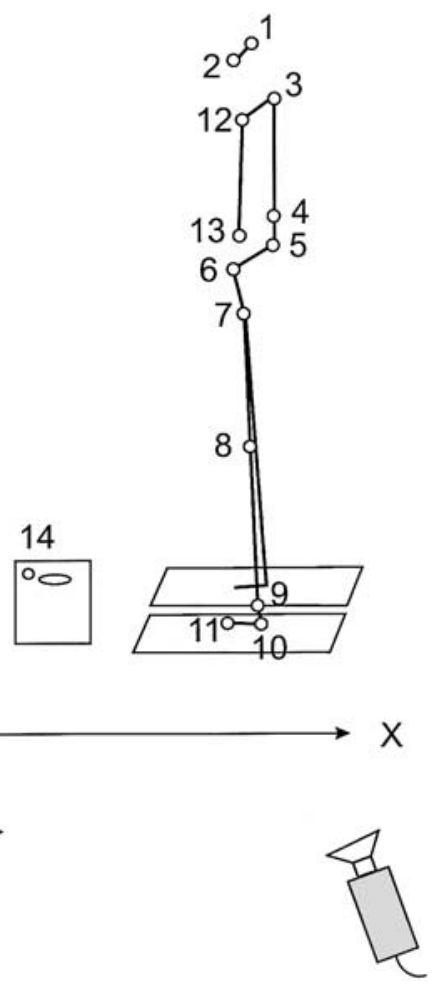

Fig 1. The experimental setup. Subject stands with each foot on a force platform, the box in front of her. Schematic shows the ELITE coordinate system and the position of the 2-movement registration cameras and the 14 light-reflecting markers. 
The relative instant of box lift-off (\% movement time in milliseconds) was calculated as ([box lift-off - onset of downward phase]/[total movement time] $) \times 100 \%$. The baseline angle was defined as the mean angle during the first $200 \mathrm{~ms}$ of recording before the auditory cue and movement onset. Lift-off angle and angular velocity are the values at the instant of box lift-off. The angular ROM was defined as the difference between the baseline angle and the absolute peak angle reached at any instant between the onset of the downward and end of the upward phases. Peak angular velocity in the down- and upward phases was taken as the absolute peak velocity reached at any instant in each of the 2 phases. The mean relative phase angle in a phase and the peak relative phase angle in a phase were calculated separately for the down- and upward phases. Because in some cases both positive and negative peaks occurred in 1 phase, the sign of the mean relative phase angle for that particular joint pair in that phase determined whether the positive or negative peak relative phase angle was considered to be the peak.

Each variable was subjected to a 1-sample KolmogorovSmirnov test to compare the observed cumulative distribution function with a theoretic normal distribution. In no case was the assumption of a normal distribution rejected. Thus, we applied independent samples $t$ tests for equality of means to determine whether variables differed between BP and NBP. We only performed a test for variables that showed a sufficiently large difference between the group means (rule of thumb: difference of group means $>$ smallest standard deviation [SD]). Each $t$ test was accompanied by the Levene test for equality of variances, and the outcome of this test determined which of the $2 t$ test output options was presented: the equal variances assumed output (with $14 d f$ ) or the equal variances not assumed output $(<14 d f)$. We report the 1-tailed significance in the test output of hypothesis 1 (duration of upward phase $\mathrm{BP}>\mathrm{NBP}$ ) and hypothesis 3 (lumbar spine flexion $\mathrm{BP}<\mathrm{NBP}$ ) and the 2-tailed significance in all other cases. We applied conventional statistics to average the relative phase angles within subjects, within groups, and within each movement phase, and to test whether mean values differed between groups. This procedure is justified if the range of relative phase angle data is not large (ie, $\left.<90^{\circ}\right),{ }^{13}$ as was the case in the present study. To identify a distal-to-proximal sequence during lifting, the percentage of negative relative phase angle values was scored for each individual trial according to McGorry and Hsiang. ${ }^{28}$ We decided that at least $66 \%$ of the values in the upward phase had to be negative to indicate a distal-to-proximal sequence. All statistical analyses were performed using SPSS for Windows, ${ }^{c}$ and in all tests differences between BP and NBP were considered significant at $P$ less than .05.

\section{RESULTS}

Despite the pain, the subjects with LBP successfully managed to perform the lifting task. Because all subjects were allowed to perform the task with a self-chosen technique and speed, differences in kinematics and speed of motion were observed. For instance, 2 subjects, 1 in each group, lifted their heels from the floor at the end of the downward and beginning of the upward phase. After the experiment, the subjects with back pain did not report a provocation of or increase in pain nor did the subjects without back pain. The time needed to perform the task did not significantly differ between the subjects with back pain (BP) and those without $(\mathrm{NBP})\left(t_{14}=.43, P=.675\right.$; fig $2)$. As hypothesized, the BP group performed the upward lifting motion more slowly than the NBP group: the duration of the upward phase was $1731 \pm 290 \mathrm{~ms}$ for BP versus $1489 \pm 187 \mathrm{~ms}$ for $\operatorname{NBP}\left(t_{14}=2.03, P=.031\right)$. Also, the relative

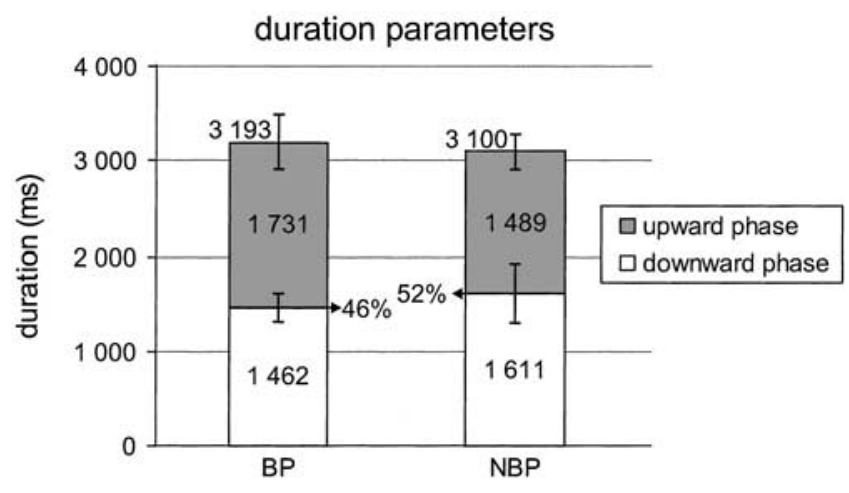

Fig 2. Mean duration (ms) of the total movement, downward phase, and upward phase for BP group $(n=7)$ and NBP group $(n=9)$. The relative instant of box lift-off is shown as a percentage of total movement time. The bars indicate the SDs for the duration of the downward and upward phases.

instant of box lift-off occurred significantly earlier for BP at $46 \% \pm 4 \%$ versus $52 \% \pm 3 \%$ of total movement time $\left(t_{14}=\right.$ $-3.12, P=.008)$. The duration of the downward phase did not differ between groups $\left(t_{14}=-1.16, P=.267\right)$.

\section{Joint Angles and Angular Velocities}

Figures 3 and 4 present plots of the angular excursions and velocities, respectively, in the BP and NBP group during the downward and subsequent upward phases. Both groups showed clear flexion-extension excursions of ankle, knee, hip, and lumbosacral joint angles, although with different ROMs. Peak flexion occurred around box lift-off, and for some joints the peak amplitude appeared to differ between groups. Statistical analyses established that the hip joint was significantly less flexed at lift-off in the BP than in the NBP group $\left(t_{14}=\right.$ $2.78, P=.015$; table 3 ). Also, the hip joint showed less flexion at baseline in $\mathrm{BP}\left(t_{14}=2.16, P=.048\right)$. The mean angular velocity time series also reflect the flexion-extension pattern in all joints (fig 4). For both BP and NBP, the average velocity at lift-off was positive in all joints, indicating that the joints were extending at that instant (table 4). The peak velocity obtained in the downward and upward phases did not differ between the 2 groups. Note that the peak velocity values in table 4 are higher than the peak values deduced from the angular velocity time series in figure 4 . This difference is because of different averaging procedures. Figure 4 presents a mean velocity time series with a peak (peak of mean) that does not necessarily coincide with the peaks of the underlying individual time series. Table 4, on the other hand, shows the mean of individual peak velocity values (mean of peaks). With respect to the timing of peak velocities, we did find 1 difference between BP and NBP. The peak extension velocity of the knee joint occurred significantly earlier in BP than in NBP: $11.6 \% \pm 2.7 \%$ versus $16.8 \% \pm 6.0 \%$ movement time after box lift-off $\left(t_{11.6}=\right.$ $-2.31, P=.040)$.

\section{Relative Phase Angles}

Figure 5 shows the relative phase angles between adjacent leg joints and between the ankle and lumbosacral joint. The expected distal-to-proximal sequence of joint rotations in the upward phase $(>66 \%$ negative values) was identified in all joint pairs of both groups. To evaluate back pain-associated differences in the timing of this sequence (hypothesis 2), group differences in mean and peak relative phase angles were ex- 

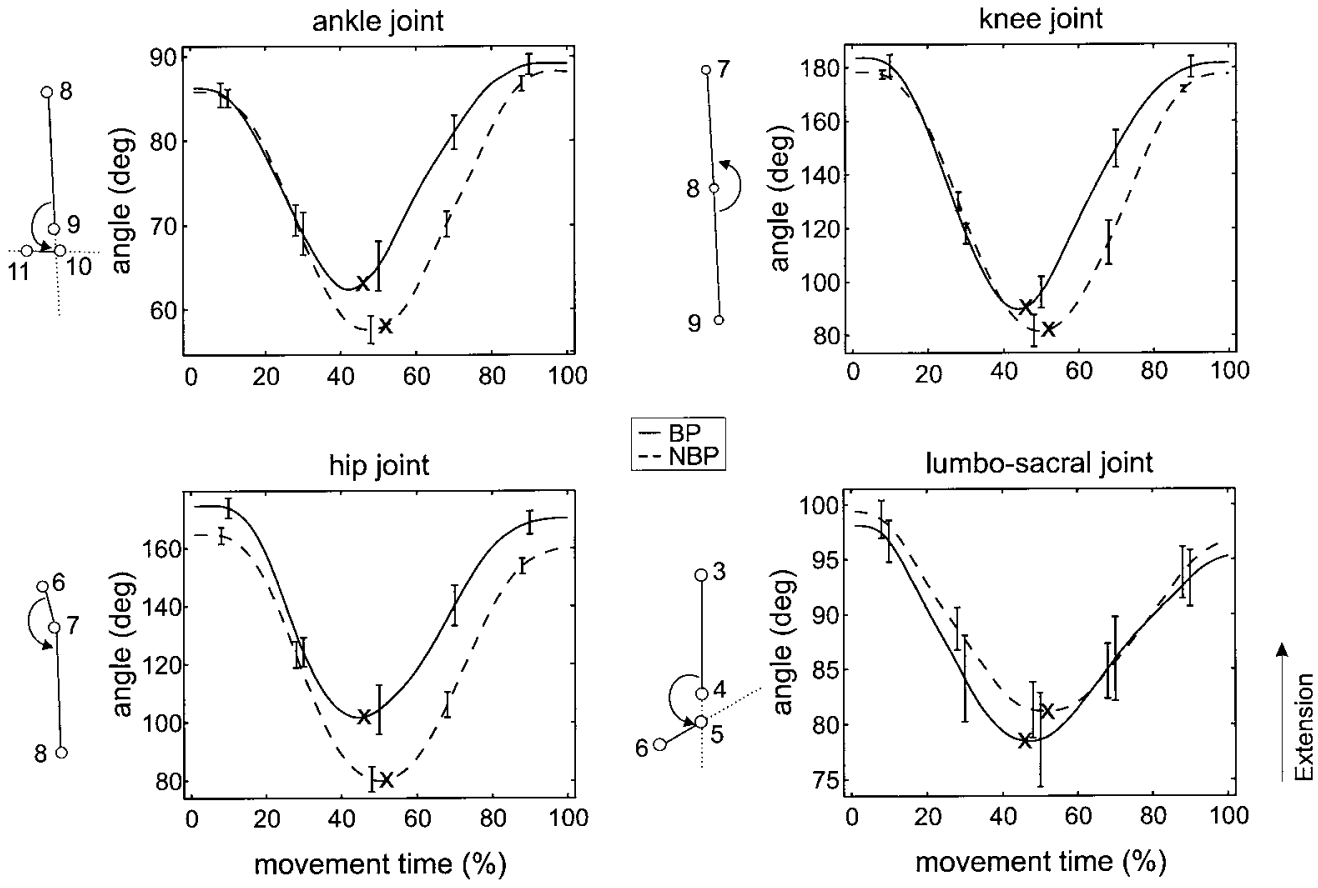

\begin{abstract}
Fig 3. Mean joint-angle time series for BP group $(n=7)$ and NBP group $(n=9)$. The bars indicate \pm 1 standard error of the mean (SEM). The $X$ marks the instant of box lift-off. NOTE. Full extension is $180^{\circ}$. full flexion is $0^{\circ}$.
\end{abstract}

amined (table 5). None of the seeming differences in mean relative phase angle reached the defined significance level. In the peak relative phase angle, we found several significant differences. First, the maximal phase lag between knee joint extension and hip joint extension was larger in the BP group $\left(t_{14}=-3.63, P=.003\right)$. Second, the peak phase lag between ankle joint flexion and lumbosacral joint flexion was oppositely directed in the downward phase $\left(t_{14}=-5.46, P<.001\right)$. For BP, the lumbosacral and ankle joints flexed in synchrony during the first third of the downward phase and after that flexion at the lumbosacral joint lagged behind flexion at the ankle joint. In the NBP group, the lumbosacral joint led the ankle joint in flexion in the first two thirds of the downward phase. In addition, the peak phase lag between both joints occurred significantly later in BP than in NBP: $28.7 \% \pm 9.5 \%$ versus $17.8 \% \pm 7.4 \%$ movement time $\left(t_{14}=2.59, P=.021\right)$.
Fig 4. Mean joint-angular-velocity time series for BP group $(n=7)$ and NBP group $(n=9)$. The bars indicate \pm 1 SEM. The $X$ marks the instant of box liftoff. NOTE. Positive values express joint extension; negative values express joint flexion.
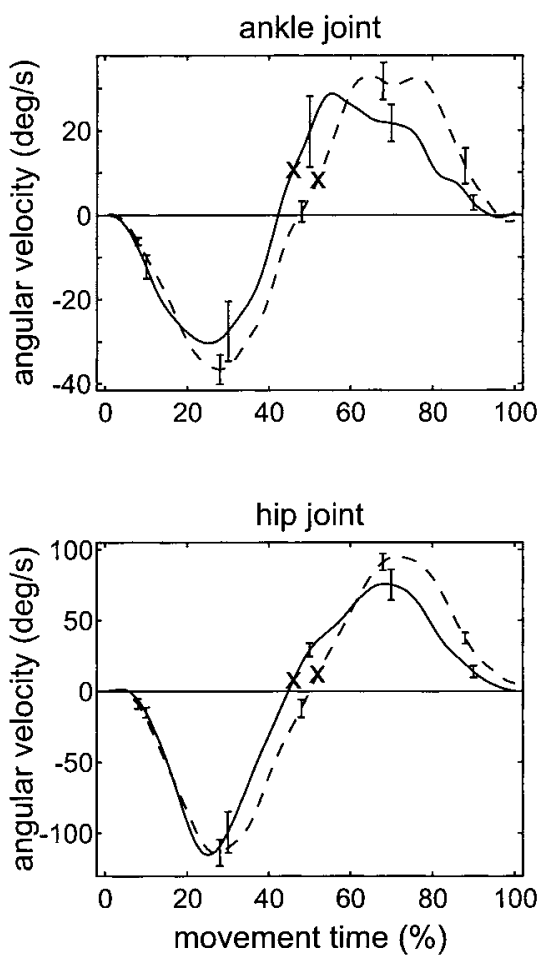
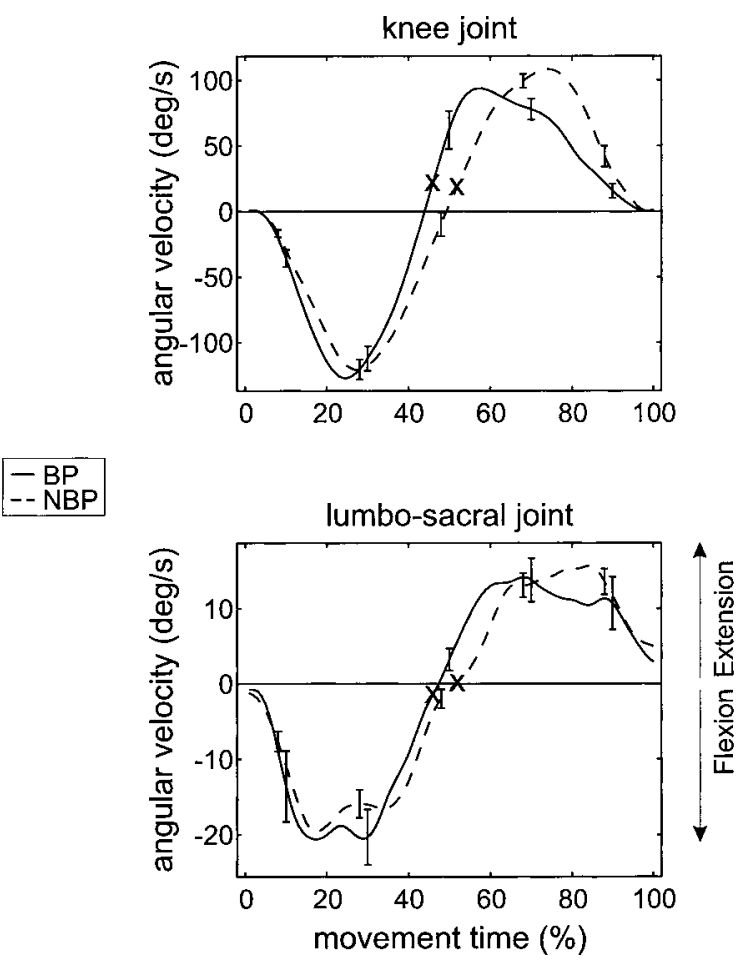
Table 3: Joint Angles at Baseline and at Box Lift-Off and Angular ROM for the BP and NBP Group

\begin{tabular}{lllll}
\hline & Ankle Joint & Knee Joint & Hip Joint & Lumbosacral Joint \\
\hline Baseline angle (deg) & & & & \\
BP & $86.3 \pm 3.1$ & $183.7 \pm 11.0$ & $174.4 \pm 9.5^{*}$ & $98.1 \pm 5.0$ \\
NBP & $85.9 \pm 4.3$ & $178.2 \pm 4.9$ & $164.6 \pm 8.5$ & $99.5 \pm 5.1$ \\
Lift-off angle (deg) & & & & $78.3 \pm 11.3$ \\
BP & $62.2 \pm 7.8$ & $89.3 \pm 8.4$ & $101.9 \pm 20.8^{*}$ & $81.0 \pm 7.7$ \\
NBP & $57.5 \pm 4.9$ & $79.9 \pm 20.8$ & $78.7 \pm 12.4$ & $20.3 \pm 8.5$ \\
ROM (deg) & & & $73.8 \pm 17.7$ & $18.8 \pm 4.3$ \\
BP & $25.1 \pm 5.9$ & $96.7 \pm 9.4$ & $86.8 \pm 12.3$ & \\
NBP & $29.2 \pm 6.1$ & $99.5 \pm 24.3$ & & \\
\hline
\end{tabular}

NOTE. Values are mean \pm SD. Full extension is $180^{\circ}$; full flexion is $0^{\circ}$.

* Difference between groups significant at $P<.05$.

\section{Pelvis and Trunk Motion}

Figure 6 presents plots of the angular excursions and velocities for the rotation of the pelvis in space and its tilt in the body (ie, relative to the trunk) and for the rotation of the trunk in space. Note that lumbar spine flexion (ie, backward pelvic tilt) is defined as positive here (lower panels), whereas flexion of the lumbosacral joint was defined as negative in figures 3 and 4 (lower right panels). The hypothesized limited lumbar spine flexion in BP subjects was not confirmed by our results; the angular ROM of the pelvis in the body was not smaller for BP than for NBP $\left(t_{14}=-1.46, P=.083\right.$; table 6$)$. On the contrary, the amount of lumbar spine flexion at lift-off was larger for BP than for NBP; the backward tilt of the pelvis in the body was larger in the first group $\left(t_{14}=2.39, P=.031\right)$. Although present at first sight, no significant differences were identified in the peak angular velocity. Note again that the peak velocity values in table 6 (mean of peaks) are higher than the peak values deduced from the angular velocity time series in figure 6 (peak of mean). The spatial pelvic angular velocity showed 2 peaks in the NBP group but multiple peaks in the BP group. The angular velocity of the pelvis segment in the body showed an immediate transition from backward tilt (ie, lumbar spine flexion) to forward tilt (ie, lumbar spine extension) at box lift-off for BP. The NBP group, however, kept the pelvis in a backward tilted position for about $20 \%$ of normalized time, implying that the lumbar spine was flexed during that period $(\sim 600 \mathrm{~ms})$.

\section{DISCUSSION}

The present study is, to our knowledge, among the first to describe differences in the coordination of lifting that are associated with pregnancy-related back pain. We have chosen, therefore, to focus on the group differences. This approach also enabled us to compare our present findings with earlier group- level data on back pain-related changes in the coordination of lifting or other trunk motions. ${ }^{20,21,24}$ Although the large withingroup variability suggests that analyzing individual lifting strategies may add some insights into the relationship between pregnancy-related back pain and task performance, these analyses are beyond the scope of the present report.

In line with our first hypothesis, the BP group required more time for the upward phase than the NBP group. Several factors may have contributed to the slower lifting motion of those with pain. First, it might be the pain itself, assigned a score of $27 \mathrm{~mm}$ on a VAS. Persons with pain tend to avoid painful motions. ${ }^{29}$ Second, the BP group reported a markedly reduced ability to lift a heavy object before the actual experiments, whereas the NBP group reported a normal ability. Fear of movement and fear of (re-)injury have been found in chronic back pain patients. ${ }^{30}$ In our experiment, this fear might have led to a conscious slowing down of the most demanding part of the task, ie, the lifting of the box. Third, back pain patients often show a reduced strength of the trunk extensor muscles ${ }^{9,31}$ and, consequently, a reduced trunk extension velocity. ${ }^{20,21}$ We did not measure trunk extensor strength in the present study. Our data suggest that the slower upward motion was not because of a slower trunk extension, because the extension velocity of the lumbosacral joint did not differ between groups. The slower upward motion can more likely be attributed to the lower extension speed of the ankle and knee joint in the decelerative part of the upward phase (see fig 4). It is not clear why BP subjects chose to slow down this part of the upward motion rather than the accelerative part. From a mechanical point of view, it is even surprising that they did because low-back loading is highest in the first part of the upward phase when body and box have to be accelerated. ${ }^{32}$

Table 4: Peak Angular Velocity in the Downward and Upward Phases and Angular Velocity at Box Lift-Off for the BP and NBP Groups

\begin{tabular}{lcccc}
\hline & Ankle Joint & Knee Joint & Hip Joint & Lumbosacral Joint \\
\hline Peak velocity down (deg/s) & & & & $-32.4 \pm 12.2$ \\
BP & $-44.8 \pm 9.2$ & $-138.6 \pm 21.8$ & $-126.8 \pm 37.7$ & $-27.4 \pm 6.1$ \\
NBP & $-47.2 \pm 10.5$ & $-139.9 \pm 15.0$ & $-129.2 \pm 32.6$ & \\
Peak velocity up (deg/s) & & & & $24.9 \pm 6.5$ \\
BP & $42.9 \pm 8.4$ & $116.8 \pm 24.1$ & $88.4 \pm 26.2$ & $23.0 \pm 5.4$ \\
NBP & $47.4 \pm 10.0$ & $124.0 \pm 23.8$ & $107.8 \pm 20.8$ & $1.4 \pm 4.3$ \\
Velocity at lift-off (deg/s) & $5.9 \pm 14.0$ & $23.4 \pm 36.3$ & $12.3 \pm 15.9$ & $0.1 \pm 3.4$ \\
BP & $7.4 \pm 6.1$ & $19.8 \pm 13.4$ & $11.9 \pm 13.2$ & \\
NBP & & & \\
\hline
\end{tabular}

NOTE. Values are mean \pm SD. Positive values express joint extension; negative values express joint flexion. 

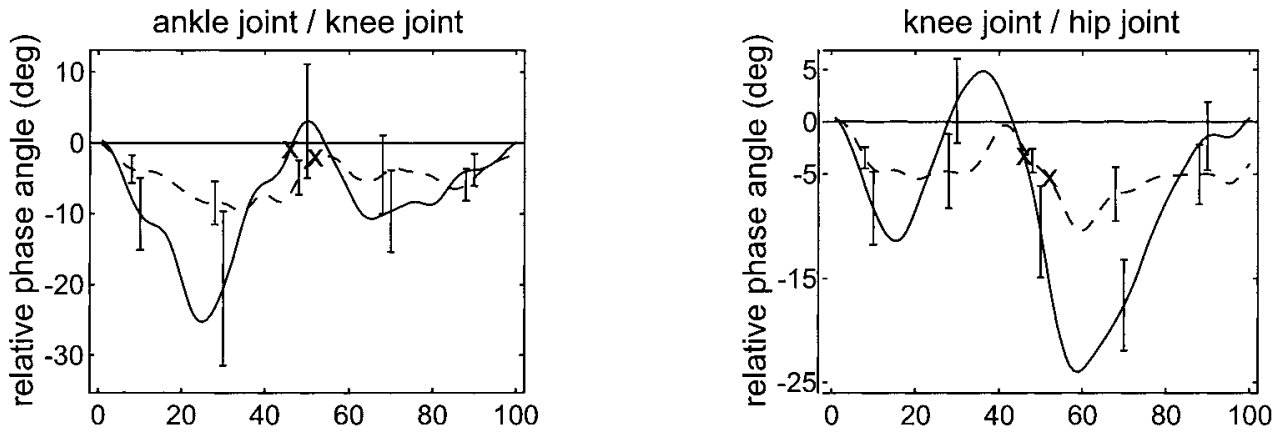

Fig 5. Mean relative-phaseangle time series for the $\mathrm{BP}$ group $(n=7)$ and NBP group $(n=9)$. The bars indicate \pm 1 SEM. The $X$ marks the instant of box lift-off. NOTE. Positive values indicate that the proximal joint led the distal one; negative values indicate that the distal led the proximal joint.

Concerning the expected back pain-associated differences in the timing of the distal-to-proximal sequence of joint rotations during the phase of lifting (hypothesis 2), we found a larger peak phase lag only between knee and hip extension. This finding implies that the knee extension of BP subjects led their hip extension to a larger extent than in NBP subjects, whereas both groups displayed the same distal-to-proximal sequence of joint rotations in the upward phase. The function of this difference, for instance with respect to the avoidance of pain or the reduction of stress, remains to be elucidated. In the phase of lowering the body toward the load, another interesting difference in joint coordination between BP and NBP was found. In the first part of this phase, the BP group seemed to put more accent on ankle flexion and less on flexion at the lumbosacral joint, the painful area, compared with the NBP group in which lumbosacral joint flexion prevailed. The function of this alternative strategy is not clear. Our data show that it did not result in a limitation of lumbar spine flexion (see fig 6), an act that would have limited the strain on sensitive structures in that area and thus pain. ${ }^{22}$ On the other hand, this strategy does facilitate forward trunk bending because active forward rotation of the whole body around the ankle joint brings the line of action of the force of gravity into a better position (ie, anterior to the line of action of the ground reaction force) to assist forward bending (as in gait initiation ${ }^{33}$ ).

Contrary to our third hypothesis, we did not find limited lumbar spine flexion in the BP subjects. Surprisingly, lumbar spine flexion at lift-off was even larger in that group. Although

Table 5: Mean Relative Phase Angle Between Joints and Peak Relative Phase Angle Between Joints in the Downward and Upward Phases for the BP and NBP Group

\begin{tabular}{|c|c|c|c|c|}
\hline & Ankle Joint/Knee Joint & Knee Joint/Hip Joint & Hip Joint/Lumbosacral Joint & Ankle Joint/Lumbosacral Joint \\
\hline \multicolumn{5}{|c|}{ Mean angle down (deg) } \\
\hline BP & $-10.8 \pm 13.3$ & $-2.4 \pm 8.3$ & $6.7 \pm 14.0$ & $-6.5 \pm 20.4$ \\
\hline NBP & $-6.0 \pm 6.8$ & $-3.4 \pm 5.2$ & $13.6 \pm 10.7$ & $4.1 \pm 12.2$ \\
\hline \multicolumn{5}{|c|}{ Mean angle up (deg) } \\
\hline BP & $-5.0 \pm 9.8$ & $-11.5 \pm 6.7$ & $-20.5 \pm 16.8$ & $-37.0 \pm 19.2$ \\
\hline NBP & $-3.8 \pm 9.4$ & $-6.6 \pm 6.6$ & $-23.0 \pm 9.9$ & $-33.4 \pm 20.1$ \\
\hline \multicolumn{5}{|c|}{ Peak angle down (deg) } \\
\hline $\mathrm{BP}$ & $-33.9 \pm 39.4$ & $-19.6 \pm 10.0$ & $34.7 \pm 17.7$ & $-42.2 \pm 38.2^{*}$ \\
\hline NBP & $-21.2 \pm 19.4$ & $-14.1 \pm 7.5$ & $46.2 \pm 19.9$ & $39.2 \pm 21.0$ \\
\hline \multicolumn{5}{|c|}{ Peak angle up (deg) } \\
\hline $\mathrm{BP}$ & $-25.5 \pm 13.5$ & $-29.7 \pm 8.3^{*}$ & $-53.8 \pm 29.3$ & $-84.5 \pm 38.9$ \\
\hline NBP & $-17.1 \pm 6.7$ & $-17.2 \pm 5.5$ & $-48.1 \pm 12.3$ & $-61.6 \pm 22.1$ \\
\hline
\end{tabular}

NOTE. Values are mean \pm SD. Positive values indicate that the proximal joint led the distal one; negative values mean that the distal joint led the proximal one.

* Difference between groups significant at $P<.05$. 
Fig 6. Mean segment-angle and segment-angular-velocity time series for BP group $(n=7)$ and NBP group $(n=9)$. The bars indicate \pm 1 SEM. The $X$ marks the instant of box liftoff. NOTE. A vertical segment orientation corresponds to $90^{\circ}$; a horizontal orientation to $0^{\circ}$. Decreasing angle and negative angular velocity values express forward tilt (pelvis) or inclination (trunk); increasing angle and positive angular velocity values express backward tilt (pelvis) or inclination (trunk).

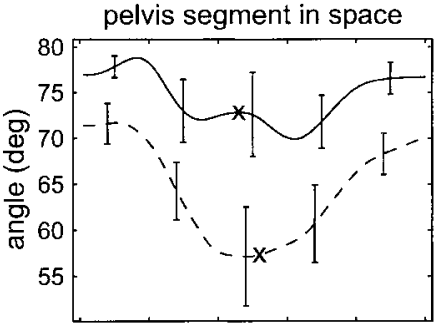

trunk segment in space
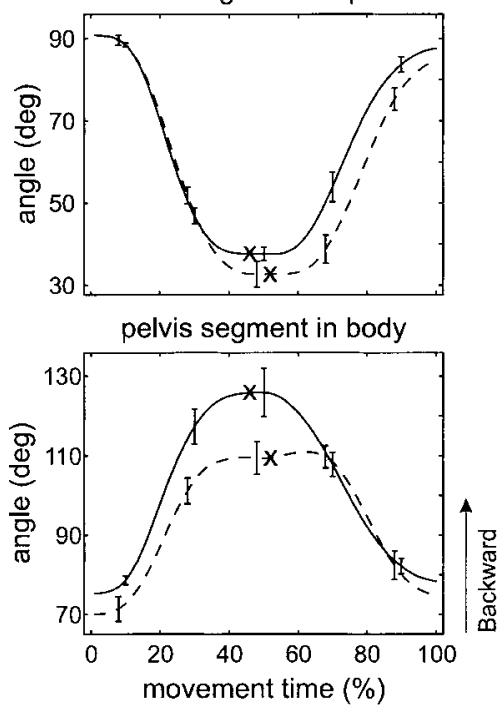

pelvis segment in space

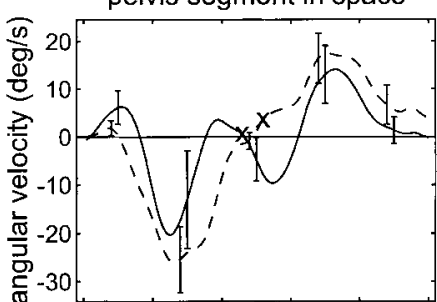

trunk segment in space

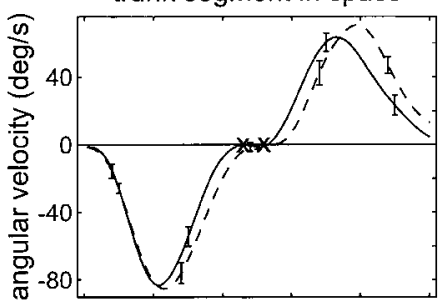

pelvis segment in body

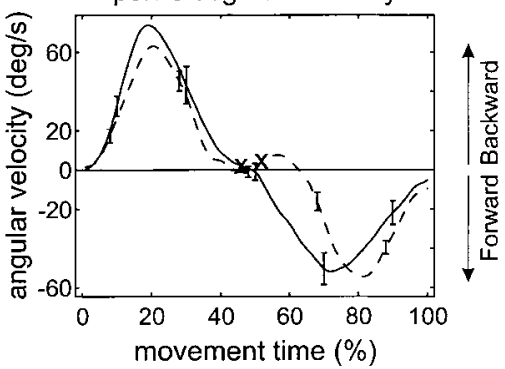

the BP subjects did not limit lumbar spine flexion, they did show an immediate transition from lumbar spine flexion to extension around lift-off, whereas the NBP group maintained the flexed lumbar spine for about $600 \mathrm{~ms}$ (see lower panels of fig 6). This immediate transition might indicate that BP subjects are unable to or prefer not to stabilize the spine in a submaximally flexed position. Picking up the load causes an increase in shear forces acting on the spine. Extension at this point will reduce these shear forces because of its effect on the muscle lines of action. ${ }^{34}$ If spinal instability underlies LBP, as has been suggested by Panjabi ${ }^{35}$ and others, ${ }^{36,37}$ these shear forces may cause excessive intervertebral translations. ${ }^{38}$ In addition, spine extension results in an increase in the moment arm of the extensor muscles with respect to the spine ${ }^{39}$ and thus

Table 6: Segment Angle at Baseline and at Box Lift-Off, Segment Angle ROM, and Peak Segment Angular Velocity in the Downward and Upward Phases for the BP and NBP Groups

\begin{tabular}{|c|c|c|c|}
\hline & Pelvis in Space & Trunk in Space & Pelvis in Body \\
\hline \multicolumn{4}{|c|}{ Baseline angle (deg) } \\
\hline BP & $76.8 \pm 3.6$ & $91.1 \pm 1.6$ & $75.0 \pm 3.3$ \\
\hline NBP & $71.6 \pm 6.7$ & $91.0 \pm 3.6$ & $70.0 \pm 9.0$ \\
\hline \multicolumn{4}{|c|}{ Lift-off angle (deg) } \\
\hline $\mathrm{BP}$ & $73.4 \pm 13.5$ & $37.8 \pm 5.0$ & $126.3 \pm 16.8^{*}$ \\
\hline NBP & $57.3 \pm 16.6$ & $33.0 \pm 10.8$ & $109.0 \pm 12.3$ \\
\hline \multicolumn{4}{|c|}{ ROM (deg) } \\
\hline $\mathrm{BP}$ & $12.6 \pm 8.7$ & $55.2 \pm 3.4$ & $-52.9 \pm 15.5$ \\
\hline NBP & $20.7 \pm 9.3$ & $60.4 \pm 9.4$ & $-43.8 \pm 9.5$ \\
\hline \multicolumn{4}{|c|}{ Peak velocity down (deg/s) } \\
\hline $\mathrm{BP}$ & $-14.3 \pm 39.2$ & $-88.3 \pm 11.8$ & $82.7 \pm 14.6$ \\
\hline NBP & $-32.6 \pm 33.7$ & $-93.9 \pm 23.4$ & $76.2 \pm 11.7$ \\
\hline \multicolumn{4}{|c|}{ Peak velocity up (deg/s) } \\
\hline $\mathrm{BP}$ & $8.9 \pm 27.9$ & $74.6 \pm 11.0$ & $-67.9 \pm 12.9$ \\
\hline NBP & $23.5 \pm 27.2$ & $79.4 \pm 19.2$ & $-64.5 \pm 12.2$ \\
\hline
\end{tabular}

NOTE. Values are mean \pm SD. A vertical segment orientation corresponds to $90^{\circ} ;$ a horizontal orientation to $0^{\circ}$. Decreasing angle and negative angular velocity values express forward pelvic tilt or trunk inclination; increasing angle and positive angular velocity values express backward pelvic tilt or trunk inclination.

* Difference between groups significant at $P<.05$. 
in a reduction of the compression forces on the lumbar spine. Hence, the fast transition to extension would be functional in BP subjects.

We still do not know whether the lifting strategy shown by the BP group represents a compensatory strategy to avoid pain and/or loading of an injured spine or whether this deviating strategy is a cause of back pain in that group. Because this is a cross-sectional study, we cannot identify cause and effect. Only a prospective study examining women without back pain before, during, and after a pregnancy can ascertain cause and effect in this case. A few observations may shed a light on this question. Because all participants performed practice trials before the actual experiments, they were able to choose an optimal lifting strategy. Pain avoidance may have been a likely optimization criterion in the BP group. ${ }^{29}$ This suggestion is supported by the fact that the $\mathrm{BP}$ subjects did not report a provocation of or increase in pain after the experiments. All subjects attended an ordinary postpregnancy exercise class and did not receive any information about good or safe lifting strategies. Finally, lifting technique (ie, stoop vs squat) does not affect low-back loading, ${ }^{39}$ suggesting that different lifting strategies do not involve differential health risks.

We conclude with a few remarks about the limitations of the current investigation. First, we have only compared BP and NBP subjects on a group level. As the large SD indicates, task performance differed considerably between subjects within each group. Further studies should search for different subgroups within a larger BP group and try to relate different types of low back and pelvic pain to different aspects of impaired lifting performance. Furthermore, the significant differences on a group level do not necessarily imply that the kinematic analysis of a load-lifting task is suitable for individual diagnostics. For instance, the $99 \%$ confidence interval of the pelvic angle in the body at lift-off in NBP included the values of 6 of $7 \mathrm{BP}$ subjects, making the parameter inapt to distinguish BP from NBP. Second, our choice to determine the onset of the downward and end of the upward phase from the time series of the knee angular velocity was arbitrary. This choice was motivated by the fact that the knee angular velocity showed a smooth bell-shaped curve with a clear deviation from zero both at the start and end of the motion, thus enabling unequivocal determination of motion onset and end. This was not possible in the time series of the angular velocity at the lumbosacral joint, which would have been a more valid indicator of the end of the lifting motion as it returned to zero after the defined end of the upward phase (see fig 4). Finally, one realizes that applying a 2-dimensional sagittal plane model with a rigid trunk segment, as we did in the present study has 2 drawbacks. Such a model will not capture motions outside the sagittal plane and differences between BP and NBP in those motions. Also, back pain-related differences in the movement pattern at the level of a single or a number of spinal vertebrae will be missed by any method that treats the trunk as a stiff segment. Such differences between BP and NBP subjects are likely to occur, because many LBP patients reported having decreased passive stiffness and increased ROM during rotation and translation of vertebrae with respect to each other. ${ }^{36,40,41}$

\section{CONCLUSION}

Although all subjects successfully executed the moderate lifting task presented to them, we found that the kinematics were indeed different in the back pain group. Most important, around the instant of lift-off their pelvic movement pattern with respect to the trunk-and consequently the change in lumbar spine curvature-appeared to be affected. This occurrence might point to a functional adaptation to prevent loading of a flexed spinal column in the back pain patients or it might be a (potentially adverse) consequence of a limited ability to stabilize the lumbar spine curvature at the instant an external load is added.

The dissimilar execution of the lifting act points to 2 potential causes of the reduced lifting ability in the back pain group, ie, sprains of posterior tissues that are stretched in flexion or insufficient muscular or passive tissue capacity to stabilize the spine. Further research is needed to determine the exact relation between the altered kinematics and the underlying disorder. Given the large variance in the lifting kinematics of the back pain group, that investigation may require a larger study population that would permit subgroup analyses.

Acknowledgments: We acknowledge the valuable assistance of Ingmarie Apel in preparing and running the experiments, and thank Petra van der Burg for critically reviewing the manuscript and Birgitta Ackelman for sharing with us her physical therapy experience with LBP patients. We greatly appreciate the expert advice on statistics of Dr. Ernest de Vroome of TNO Work and Employment.

1. Mantle MJ, Greenwood RM, Currey HL. Backache in pregnancy. Rheum Rehabil 1977;16:95-101.

2. Östgaard HC, Andersson GB, Karlsson K. Prevalence of back pain in pregnancy. Spine 1991;16:549-52.

3. Kristiansson P, Svärdsudd K, von Schoultz B. Back pain during pregnancy; a prospective study. Spine 1996;21:702-9.

4. Östgaard HC, Andersson GB. Postpartum low-back pain. Spine 1992; 17:53-5.

5. Nilsson-Wikmar L, Harms-Ringdahl K, Pilo C, Pahlbäck M. Back pain in women post-partum is not a unitary concept. Physiother Res Int 1999;4:201-13.

6. Östgaard HC. Assessment and treatment of low back pain in working pregnant women. Semin Perinatol 1996;20:61-9.

7. Luoto S, Taimela S, Hurri H, Aalto H, Pyykkö I, Alaranta H. Psychomotor speed and postural control in chronic low-back pain patients; a controlled follow-up study. Spine 1996;21:2621-7.

8. Magnusson ML, Aleksiev A, Wilder DG, et al. Unexpected load and asymmetric posture as etiologic factors in low back pain. Eur Spine J 1996;5:23-35.

9. Lee JH, Ooi Y, Nakamura K. Measurement of muscle strength of the trunk and the lower-extremities in subjects with history of low-back pain. Spine 1995;20:1994-6.

10. Roy SH, De Luca CJ, Casavant DA. Lumbar muscle fatigue and chronic lower back pain. Spine 1989;14:992-1001.

11. Takemasa R, Yamamoto H, Tani T. Trunk muscle strength in and effect of trunk muscle exercises for patients with chronic low-back pain; the differences in patients with and without organic lumbar lesions. Spine 1995;20:2522-30.

12. Mens JM, Vleeming A, Stoeckart R, Stam HJ, Snijders CJ. Understanding peripartum pelvic pain; implications of a patient survey. Spine 1996;21:1363-70.

13. Burgess-Limerick R, Abernethy B, Neal RJ. Relative phase quantifies interjoint coordination. J Biomech 1993;26:91-4.

14. van Dieën JH, Toussaint HM, Maurice C, Mientjes M. Fatiguerelated changes in the coordination of lifting and their effect on low back load. J Mot Behav 1996;28:304-14.

15. Scholz JP. Organizational principles for the coordination of lifting. Hum Mov Sci 1993;12:537-76.

16. Scholz JP, Millford JP, McMillan AG. Neuromuscular coordination of squat lifting. I: Effect of load magnitude. Phys Ther 1995; 75:119-32.

17. van Dieën JH, van der Burg P, Raaijmakers TA, Toussaint HM. Effects of repetitive lifting on kinematics: inadequate anticipatory control or adaptive changes? J Mot Behav 1998;30:20-32.

18. Sparto PJ, Parnianpour M, Reinsel TE, Simon S. The effect of fatigue on multijoint kinematics, coordination, and postural stability during a repetitive lifting test. J Orthop Sports Phys Ther 1997;25:3-12.

19. Lindbeck L, Kjellberg K. Gender differences in lifting technique. Ergonomics 2001;44:202-14. 
20. Shirado O, Ito T, Kaneda K, Strax TE. Flexion-relaxation phenomenon in the back muscles; a comparative study between healthy subjects and patients with chronic low back pain. Am J Phys Med Rehabil 1995;74:139-44.

21. Szpalski M, Michel F, Hayez J-P. Determination of trunk motion patterns associated with permanent or transient stenosis of the lumbar spine. Eur Spine J 1996;5:332-7.

22. Besson JM. The neurobiology of pain. Lancet 1999;353(9164): $1610-5$.

23. Harms-Ringdahl K, Brodin H, Eklund L, Borg C. Discomfort and pain from loaded passive joint structures. Scand J Rehabil Med 1983;15:205-11.

24. Larivière C, Gagnon D, Loisel P. The effect of load on the coordination of the trunk for subjects with and without chronic low back pain during flexion-extension and lateral bending tasks. Clin Biomech 2000;15:407-16.

25. Salén B, Spangfort E, Nygren Å, Nordemar R. The Disability Rating Index: an instrument for the assessment of disability in clinical settings. J Clin Epidemiol 1994;47:1423-35.

26. Ferrigno G, Pedotti A. ELITE: a digital dedicated hardware system for movement analysis via real-time TV signal processing. IEEE Trans Biomed Eng 1985;32:943-50.

27. Kelso JA, Saltzman EL, Tuller B. The dynamical perspective on speech production. J Phonetics 1986;14:29-59.

28. McGorry RW, Hsiang SM. The effect of industrial back belts and breathing technique on trunk and pelvic coordination during a lifting task. Spine 1999;24:1124-30.

29. Boston JR, Rudy TE, Lieber SJ, Stacey BR. Measuring treatment effects on repetitive lifting for patients with chronic low back pain: speed, style, and coordination. J Spinal Disord 1995;8:34251.

30. Vlaeyen JW, Kole-Snijders AM, Boeren RG, van Eek H. Fear of movement/(re)injury in chronic low back pain and its relation to behavioral performance. Pain 1995;62:363-72.

31. Mayer TG, Smith SS, Keeley J, Mooney V. Quantification of lumbar function. Part 2: Sagittal plane trunk strength in chronic low-back pain patients. Spine 1985;10:765-72.
32. de Looze MP, Toussaint HM, van Dieën JH, Kemper HC. Joint moments and muscle activity in the lower extremities and lower back in lifting and lowering tasks. J Biomech 1993;26:1067-76.

33. Lepers R, Brenière Y. The role of anticipatory postural adjustments and gravity in gait initiation. Exp Brain Res 1995;107:11824

34. Macintosh JE, Bogduk N, Pearcy MJ. The effects of flexion on the geometry and actions of the lumbar erector spinae. Spine 1993; 18:884-93.

35. Panjabi MM. The stabilizing system of the spine. Part I. Function, dysfunction, and enhancement. J Spinal Dis 1992;5:383-9.

36. Gertzbein SD. Segmental instability of the lumbar spine: in vitro and in vivo studies. Semin Spine Surg 1991;3:130-5.

37. Sihvonen T, Partanen J, Hänninen $O$, Soimakallio S. Electric behavior of low back muscles during lumbar pelvic rhythm in low back pain patients and healthy controls. Arch Phys Med Rehabil 1991;72:1080-7.

38. Posner I, White AA, Edwards WT, Hayes WC. A biomechanical analysis of the clinical stability of the lumbar and lumbosacral spine. Spine 1982;7:374-89.

39. Tveit P, Daggfeldt K, Hetland S, Thorstensson A. Erector spinae lever arm length variations with changes in spinal curvature. Spine 1994;19:199-204.

40. LaRocca H. Segmental spinal instability. Evolution of a concept. Semin Spine Surg 1991;3:94-104.

41. Schmidt TA, An HS, Lim TH, Nowicki BH, Haughton VM. The stiffness of lumbar spinal motion segments with a high-intensity zone in the anulus fibrosus. Spine 1998;23:2167-73.

\section{Suppliers}

a. ELITE motion analyzer; BTS SpA, Via C. Colombo, 1A, 20094 Corsico Milan, Italy.

b. MATLAB ${ }^{\circledR}$ for Windows, version 4.0; The MathWorks Inc, 3 Apple Hill Dr, Natick, MA 01760-2098.

c. Release 7.5.2; SPSS Inc, 233 S Wacker Dr, 11th Fl, Chicago, IL 60606 